\title{
Cascade failure analysis of power grid using new load distribution law and node removal rule
}

\author{
Hai-Peng Ren • Jihong Song • Rong Yang • Murilo S Baptista - Celso \\ Grebogi
}

Received: date / Accepted: date

\begin{abstract}
The power grid is a directional complex network of generators, substations, and consumers. We propose a new load distribution law to emulate the power grid. We assume that the power flow is transferred through all the paths connecting generators and consumers according to their efficiency. The initial generation of generators and the initial loads of substations are calculated according to the path efficiency and the load of the consumers. If a node fails, it is removed from the power grid, and all paths passing through it will fail to transfer power. In that case, the loads of the corresponding consumers are redistributed within the whole network. During the failure cascading and propagation procedure, our node removal rule is to remove
\end{abstract}

Hai-Peng Ren

Shaanxi Key Laboratory of CSCIIP, Xian University of Technology, Xian, 710048, China

E-mail: renhaipeng@xaut.edu.cn

Jihong Song

Department of Information and Control, Xian University of Technology, Xian, 710048, China

E-mail: sjh5319693@126.com

Rong Yang

Department of Information and Control, Xian University of Technology, Xian, 710048, China

E-mail: 751296271@qq.com

Murilo S Baptista

Institute for Complex System and Mathematical Biology, University of Aberdeen, King's College, Aberdeen, AB24 3UE, UK

E-mail: murilo.baptista@abdn.ac.uk

Celso Grebogi

Institute for Complex System and Mathematical Biology, University of Aberdeen, King's College, Aberdeen, AB24 3UE, UK

E-mail: grebogi@abdn.ac.uk the first overload node along the opposite direction of power flow, then the network distributes load and goes on the cascade procedure. Our new removal rule for nodes does suppress the large scale cascading failures. This work would be very helpful for designing the protective relay system and the tolerant parameters of the grid.

Keywords Cascade failure - Load distribution law · Node removal rule · Node capacity

\section{Introduction}

Our lives are filled with an increasing variety of networks, such as transportation networks, internet, power grids, and communication networks. In those network$\mathrm{s}$, cascading failure means that one or several nodes or edges failure leads to failure of other nodes. Cascading failure might ultimately lead to the failure of a considerable number of nodes or the entire network.

Over the past decades, research on cascading failure has become an important topic in the theory of complex networks [1]. Several different models of cascading failure have been proposed. These models can be roughly divided into two categories. In the first category, only the topology of the network is considered, and the dynamics and properties of the nodes are neglected [2-22]. In the second category, both the dynamics of the nodes and the topology of the network are considered [23-25].

Cascading failure was investigated in a congested complex network $[2,3]$. In this model, the congestion effect, defined as link performance function or cost function, maps link flow to travel time. The node failure results in longer traveling times, while the failure does not affect the topology of the network. Wu et al. proposed a model to study the cascading failure triggered by link 
congestion $[4,5]$. Wu et al. also considered a capacity and a load-generating rate in traffic network [6]. The models in [2-6] are often used for analyzing cascading failures of transportation and communication networks.

Another model was proposed to measure the information transfer and storage taking place in the network during the cascading process $[7,8]$. Wang et al $[9]$ proposed that the initial load of a edge was a function of the degrees of the nodes. When the load on a edge exceeds its capacity, it was not removed from the network, but it could assign the extra load to its neighboring edges. Kinney et al. [10] modelled the cascade process in the power grid, dividing the nodes of the power grid into three groups: the generators, the substations and the distribution centres. The capacity and load of a node are calculated without considering the differences of the node types. The assumption that node failure does not affect the topology of the network in [2-10] is different from that in the actual power grids.

Wang et al. [11] assumed that the load of a node was its betweenness centrality (BC), which was defined as the total number of shortest paths crossing the node. The capacity of a node was defined, using two control parameters, as a monotonically increasing function of its initial load. Li et al. [12] assumed that the load of a node or edge is the number of shortest paths through the node or edge. Lai et al. [13] simplified the capacity function in [11] by eliminating one control parameter. However, the assumption in [11] that the energy transfers only through the shortest path is not applicable to many cases, such as power grid and transportation network. The models in [14-16] proposed that the load of a failed node was redistributed to its neighbouring nodes. The models in [14-16] assume that the load of a generator was only determined by its degree is of no practical significance to a power grid.

Wang et al. [17-19] proposed that the initial load of a node was a function of the product of its degree and the sum of its neighbouring nodes degrees. They assumed that the load of a failure node was reassigned to its neighbouring nodes. Wang et al. [20] studied the cascading failures caused by the failure of edges using the Ohms law and the Kirchhoff conservation law. Bao et al. [21] introduced a model in which an admittance matrix was defined and the DC power flow approximation was used. Simonsen et al. [22] proposed that the load of each edge was the sum of the flows that passed this edge in both directions. Although the models [20-22] are closer to the practical situation of a power grid, the calculation is complicated.

Another category of model about cascading failures considers the dynamics of nodes. Filatrella et al. [23] demonstrated that the power grid could be modelled by the interconnected Kuramoto-like model. Carareto et al. [24] extended the work in [23] to understand what would be the condition for a power grid modeled by the Kuramoto-like network to present stable frequency synchronization. The nodes in the models in [23,24] were divided into the generator and the consumer with identical dynamical equation. Sakaguchi and Matsuo [25] proposed different dynamical equation for the generator and the consumer. This category model considered both dynamics of the nodes and their synchronization. It provides a more realistic physical description, but demands a lot of computation cost to predict the redistribution of loads.

In this work, a new model for studying cascading failure in the power grid is proposed. The nodes in our proposed model are divided into three categories: the generator, the substation, and the consumer. The initial loads of consumers are predetermined, the initial generation of generators and the initial loads of substations are calculated according to the efficiencies of the paths and the load of consumers. The distribution of the initial loads satisfies the energy balance law in the power grid, i.e., the sum of the generators loads minus the sum of the energy lost on the paths (transmission line) is equal to the sum of the consumers loads. In our proposed model, the load is transferred directionally through all the paths from generators to consumers. The higher the efficiency of a path, the greater the power flow transferred by the path. If a node fails, the node is removed from the power grid, the consumers have to get the power through other paths excluding the failed node and then the loads of nodes on these paths will become larger. If there are some nodes whose loads exceed their capacities on these paths, we remove the first overloaded node along the opposite direction of power flow. We repeat the above mentioned process, until loads of all the remaining nodes are lower than their capacities. We do not consider the dynamic state of the node, so we do not need to solve the differential equations to calculate the phase and frequency of the node, which simplifies the calculation process.

The cascading failure model in this paper has the following features. Firstly, the power grid is modelled as a directed complex network consisting of generators, substations and consumers. Secondly, the power follows from the generators to the consumers through all the paths connecting them, the amount of power transferred depends on the efficiency of the path. Thirdly, the first overload node is removed along the reverse direction of the power flow, then the power flow is recalculated according to the new topology. All these features of the proposed model are consistent with the practical situation. For the best knowledge of authors, this model 
is being proposed for the first time. Our simulation results show that, under the proposed load redistribution law and the new node removal rule, the rate of cascading failure is much less than those predicted by some existing models.

\section{The proposed load distribution law}

In our proposed model, the nodes of the power grid are divided into three categories: generators, substations, and consumers. The power that node $i$ delivers to node $j(i \neq j)$ includes the power dissipated on the edge $(i, j)$ due to the resistance of the power line and the power that node $j$ receives. So, we define the edge efficiency $a_{i j}$ as the ratio between the power that node $j$ receives from node $i$ and the total power that node $i$ transmits to node $j$. If the power flow is transferred from node $i$ to node $j$, the edge efficiency $a_{i j}$ has a value in $(0,1]$; otherwise, $a_{i j}=0$. We can then get the efficiency matrix $A=\left\{a_{i j}\right\}$. We define a path efficiency matrix $E$; the element of $E$, i.e., $e_{i j}$, is a value which stores the efficiency information of all the paths from generator $i$ to consumer $j$. In the power network, if there is an edge between nodes $i$ and $j$, we define the weight of the edge as the absolute value of the admittance between nodes $i$ and $j$, otherwise, it is 0 . Then, the path efficiency between two nodes connected by a path can be calculated from the harmonic composition of weights of all edges along this path. The harmonic composition of $n$ edges with admittances $x_{1}, x_{2}, \ldots, x_{n}$ on a path is given as

$$
\left\{\sum_{i=1}^{n}\left(1 / x_{i}\right)\right\}^{-1}
$$

Therefore, given a real power grid network as that in [26], we can calculate the efficiency matrix.

Here, we assume that there is no generator located in the path from a generator to a consumer, and the substation is only responsible for the distribution of load and does not consume power. The capacity of a node is the maximum load that the node can handle; we assume that the capacity of a node is proportional to its initial load determined by the tolerant parameter $C_{c}$. We define the efficiency of a path as the product of the efficiencies of all edges in the path.

The proposed Load Distribution Law (LDL) is given as follows:

Figure 1(a) shows the connection relationship between generators and consumer $j$, and Fig. 1(b) shows the connection relationship between consumers and generator $i$. The arrow in Fig. 1 represents the direction of power flow. We assign a initial load to each consumer. According to the path efficiency matrix $E$, we can get

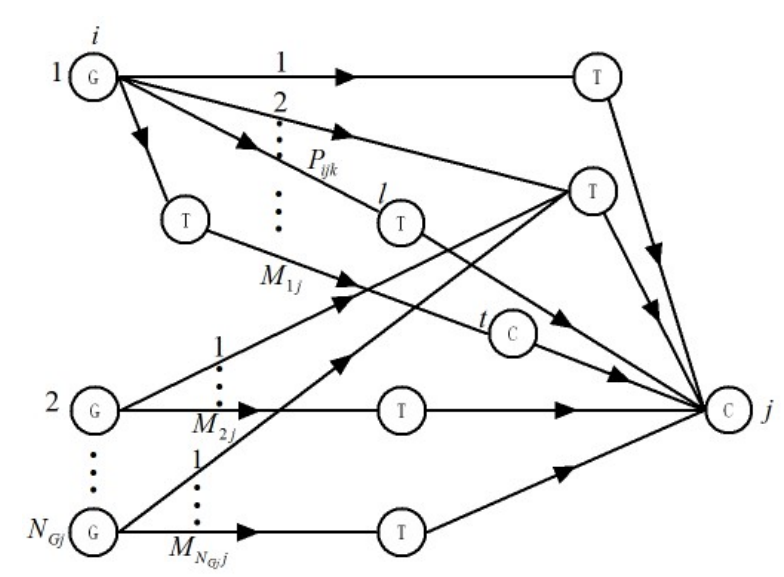

(a)

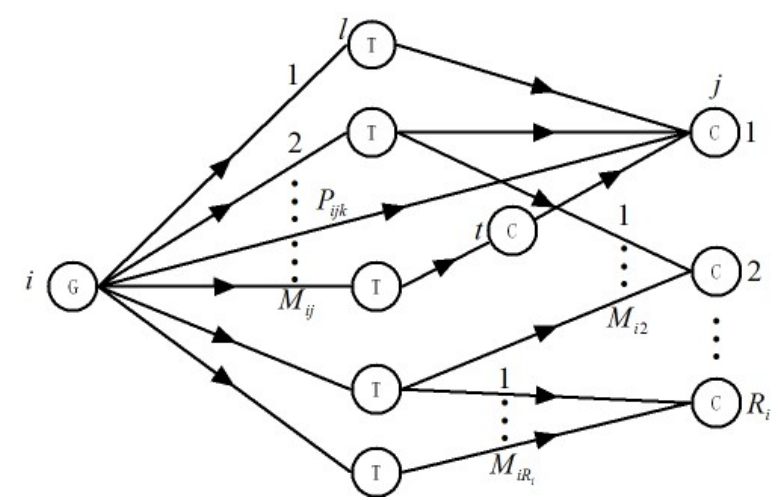

(b)

Fig. 1 Illustration of (a) the paths connecting generators represented by $\mathrm{G}$ to consumer $j$ and (b) paths connecting generator $i$ to the consumers

the efficiency of every path connecting consumer $j$ with generator $i$. Define $P_{i j k}$ as the $k$ th path connecting generator $i$ and consumer $j, e_{i j k}$ as the efficiency of path $P_{i j k}, L_{C j}$ as the load of consumer $j$. Then we can calculate the contribution to the load of consumer $j$ coming from generator $i$ through the $k$ th path given as

$$
L_{G i j k}=\frac{e_{i j k}}{\sum_{m=1}^{N_{G j}} \sum_{n=1}^{M_{i j}} e_{m n k}} L_{C_{j}}
$$

where $M_{i j}$ is the number of paths connecting generator $i$ to consumer $j, N_{G j}$ is the number of generators that have path with consumer $j$ as shown in Fig. 1(a).

Considering the efficiency of the edge, the load of generator $i$ including the load supplied to consumer $j$ and the corresponding path loss is calculated as

$L_{G i j}=\sum_{k=1}^{M_{i j}} \frac{L_{G i j k}}{e_{i j k}}$

The load of generator $i$ is given as

$L_{G i}=\sum_{j=1}^{R_{i}} L_{G i j}$ 
where $R_{i}$ is the number of consumers that have path with generator $i$ as shown in Fig. 1(b).

We define $e_{i j k l}$ as the efficiency from generator $i$ to substation $l$ on the path $P_{i j k}$, as shown in Fig. 1(a), and it can be calculated by the product of the edge efficiency from generator $i$ to substation $l$. If the substation $l$ does not exist on the path $P_{i j k}, e_{i j k l}=0$. Thus we can get the load of substation $l$ as

$L_{T l}=\sum_{i=1}^{N_{i}} \sum_{j=1}^{R_{i}} \sum_{k=1}^{M_{i j}} \frac{L_{G i j k}}{e_{i j k}} e_{i j k l}$

where $N_{i}$ is the number of generators in the power grid.

We define $e_{i j k t}$ as the efficiency of the path from generator $i$ to consumer $t$ on the path $P_{i j k}$ as shown in Fig. 1(a). It can be calculated by the product of the edge efficiency from generator $i$ to consumer $t$. $e_{i j k t}=0$, if consumer $t$ does not exist on the path $P_{i j k}$. If consumer $t$ is located on the path $P_{i j k}$, the load of consumer $t$ is calculated as

$L_{F C t}=L_{C t}+\sum_{t=1}^{N_{i}} \sum_{j=1, j \neq t}^{R_{i}} \sum_{k=1}^{M_{i j}} \frac{L_{G_{i j k}}}{e_{i j k}} e_{i j k t}$

where $L_{C t}$ is the power consumed by node $t$ itself, and the second term is the load flowing from consumer $t$ to consumer $j$. The capacity of node $i$ is defined as

$C_{i}=C_{c} L_{i}$

where $C_{c} \geq 1$ is the tolerant parameter, $L_{i}$ could be $L_{G i}, L_{T i}$ or $L_{F C i}$, according to the type of node.

\section{The proposed node removal rule in cascade process}

In this model, we consider the possible cascading failures caused by the different types of nodes. If a node in the power grid fails, the node is removed from the grid, causing matrix $A$ and matrix $E$ to change accordingly. Thus the load of the remaining nodes are redistributed. The load redistribution procedure is given by Eqs.(1) to (5). According to the procedure, one node failure can induce other nodes failure, leading to a cascading process, until the loads of all remaining nodes in the network are lower than their capacities.

In order to avoid large scale cascading failure happening, we propose a new node removal rule. If a node in the grid fails, it is removed from the grid. The load passing through this node will be delivered by other paths to the ultimate consumers; it can lead the nodes on other paths to be overload. If along a path connecting a generator to the ultimate consumer, there are more than one overload nodes, we just remove the first overloaded node along the opposite direction of

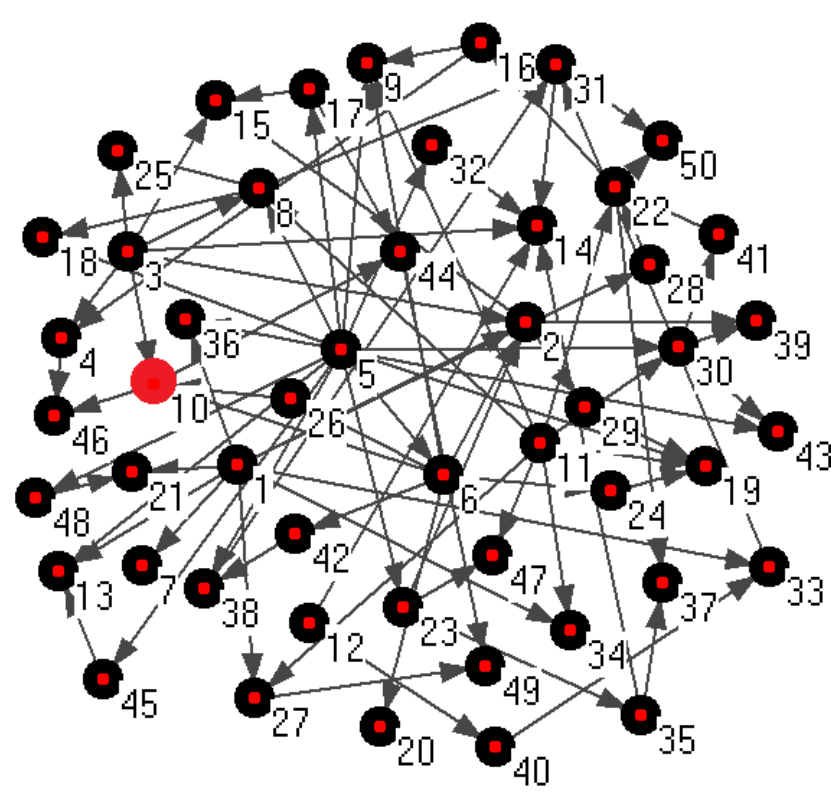

Fig. 2 Topology of our scale-free network

power flow and keep the other overload nodes on the paths, then recalculate the load until loads of all the remaining nodes are below their capacities. Under this node removal rule, cascading process will be terminated rapidly and the scale of cascading failure is suppressed. This node removal rule can be implemented by setting the parameters of the protecting relay system of power grid $[27,28]$, therefore, it is of practical significance.

In order to facilitate analysis, we define the normalized avalanche size [17] as

$g=G /(N-1)$

where $N$ is the total number of nodes in the power grid, $G$ is the total number of the failed nodes.

\section{Simulation results}

\subsection{Demonstration of the proposed node removal rule}

In order to demonstrate the proposed node removal rule in detail, we analyse the cascading failure caused by consumer 10 in a directional scale-free network consisting of 50 nodes. The topology of the scale free network is given in Fig. 2. We divide the nodes into the three categories: generators, substations and consumers. We assume that nodes $1,3,5,11,22$ are generators, nodes $2,6,8,23,30$ are substations and the remaining nodes are consumers. Assume the $C_{c}=1$, the failure caused by failed consumer 10 is given as follows.

Figure 3 shows a part of the network topology related to consumer 10. We can see from Fig. 3 that consumers 44, 46, and 49 get power from generators 3 and 


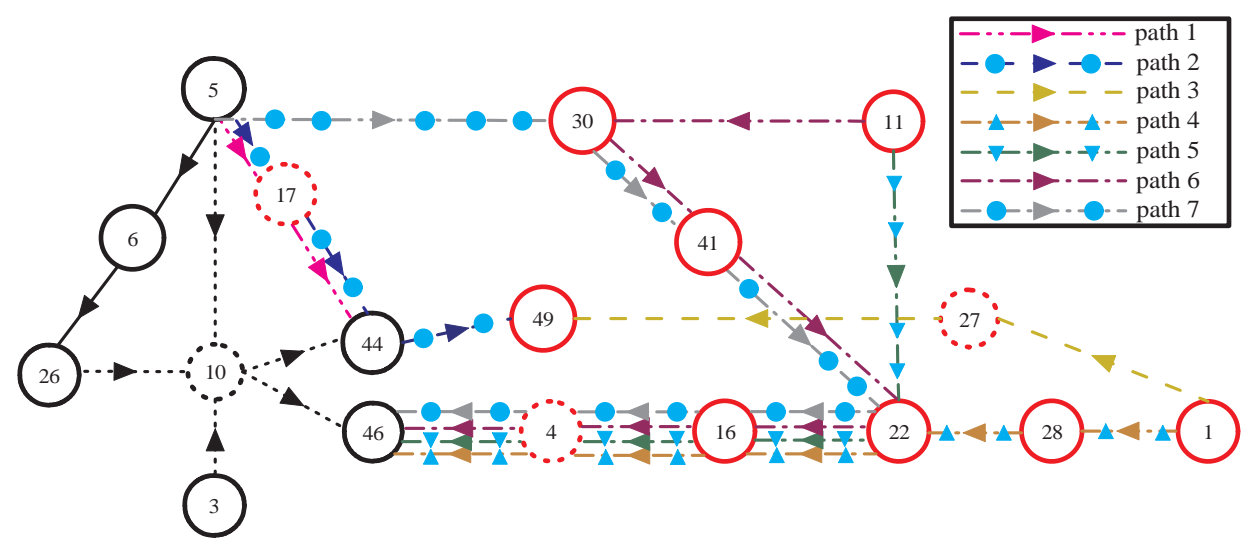

Fig. 3 Part of network topology related to consumer 10 of the scale-free network

5, through the paths passing consumer 10. If consumer 10 is removed from the network, the paths would fail to transfer power from generators 3 and 5 to consumers 44,46 , and 49 . Then the power consumed by the nodes 44,46 , and 49 has to be delivered from other paths that connect the generators to these nodes. When we check the paths that connect 44 with generators, we find a path from generator 5 to consumer 44 via node 17. When we check the paths that connect node 49 with generators, we find two paths: one is the path from generator 5 to consumer 49 via nodes 17 and 44; the other is the path from generator 1 to consumer 49 via node 27. Similarly, we can find four paths connecting generators 1, 5 and 11 to consumer 46. All these pathes are shown by the lines with different colors and marks in Fig.3. According to the efficiency matrix and the load distribution law given in Eqs. (2)-(6), we calculate the load of each node on the total seven paths. We find that the nodes on these paths are all over load when $C_{c}=1$. According the commonly used node removal rule, all these nodes including $17 ; 27,1 ; 4,16,22,28,11,41$, 30 should be removed, then, according to the load distribution law, the power transmission is recalculated. But in our proposed node removal rule, we only remove nodes 4, 17, 27 in this step, which are the first overload nodes on the paths connecting generators to consumers 44, 46 and 49 towards the opposite direction of power flow. Then the load redistribution law is used to redistribute the load, and continue this procedure. In case of node 10 failure, using our proposed node removal rule, only 9 nodes is broken down finally. But if we use the commonly used node removal rule, only 16 nodes can still work finally.

From the above example, we have verified that our proposed node removal rule can reduce the avalanche size of the cascade failure, because the first overloaded node is firstly cut rather than many others along the path being cut. This node removal rule can be im- plemented in power network by setting different relay protection time to different priority nodes.

\subsection{Cascade failure analysis of scale free network}

In this subsection, the proposed load distribution law and node removal rule are used to simulate the scale free network given in Fig. 2.

We first simulate the cascading failures triggered by the failed generators. The avalanche size $g$ versus the tolerant parameter $C_{c}$ triggered by the failures of generator 3 and 22 are shown in Fig. 4(a) and Fig. 4(b), respectively. From Fig. 4, we see that, the $g$, as a function of $C_{c}$, exhibits a non-monotonic property. In Fig. $4(\mathrm{a})$, if $C_{c}=1$, the first overload node is node 4 , which has a degree value equal to 3 . But for the same topology, if $C_{c}=1.1$, the first overload node is node 6 , which has a degree equal to 8 . It can be imagined that the final avalanche size for $C_{c}=1$ and $C_{c}=1.1$ might be quite different even though $C_{c}=1.1$ is bigger than $C_{c}=1$, so, the $g$ for $C_{c}=1.1$ is larger than that for $C_{c}=1$. When $C_{c}$ is greater than 1.3 for Fig. 4(a), the generator failure does not cause cascading failure. While, when generator 22 fails, there is no path connecting consumer 16 to get power from any other generators and that makes the avalanche size $g$ will never be 0 , no matter how large the $C_{c}$ is.

The cascading failures caused by substations are shown in Fig. 5. Figures 5(a) and (b) are the plot of $g$ versus $C_{c}$ triggered by the failures of substation 8 and 23, respectively. From Fig. 5, we see that, with the increase of $C_{c}$, the avalanche size $g$ decreases. In Fig. $5(\mathrm{a})$, when $C_{c}$ is greater than 1.3 , the substation failure does not cause cascading failure, so the avalanche size is 0 . When substation 23 fails, there is no path connecting consumer 35 to get power from any generators. So, the 


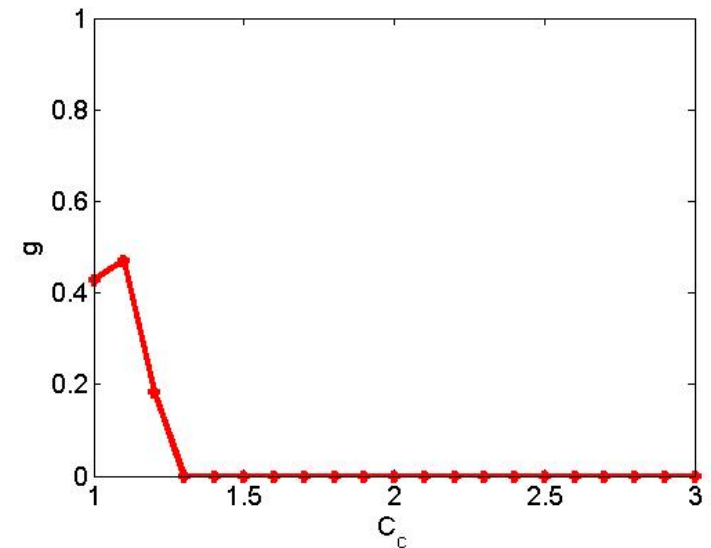

(a)

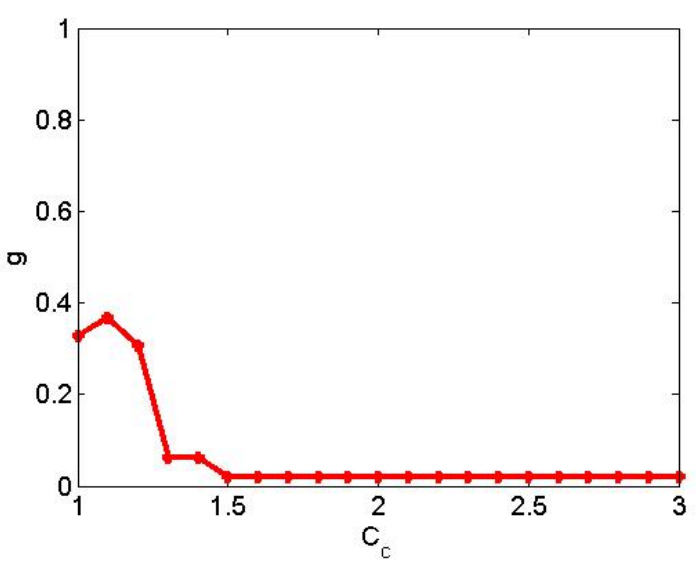

(b)

Fig. 4 Cascading failure triggered by generator 3 and 22 failure in the scale-free network is shown in subplot(a) and (b), respectively

avalanche size $g$ in Fig. 5(b) will never be 0 , no matter how large the $C_{c}$ is.

Figure 6 shows the cascading failures triggered by consumer 10 and 31, respectively. From Fig. 6(a), we see that with the increase of $C_{c}$, the avalanche size $g$ decreases. While, in Fig. 6(b), the $g$, as a function of $C_{c}$, exhibits a non-monotonic property. When $C_{c}$ is greater than 1.6 for Fig. 6(a) and 1.2 for Fig. 6(b), the consumer failure does not cause cascading failure.

\subsection{Cascade failure analysis of UK power grid}

Next, we use the UK power grid [29] consisting of 120 nodes and 165 edges. The topology of UK power grid is given in Fig. 7. By assuming that nodes 17, 21, 25, 37, $41,60,68,71,77,83,93,111,120$ are generators, nodes $18,23,46,55,65,70,85,92,94,100$ are substations

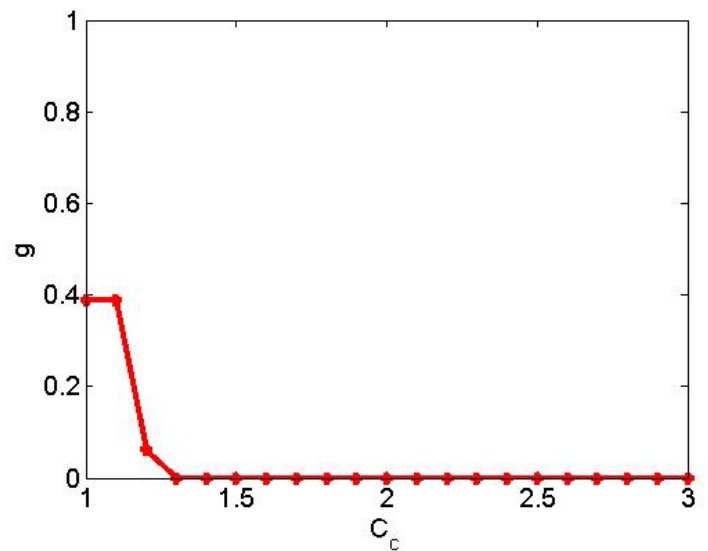

(a)

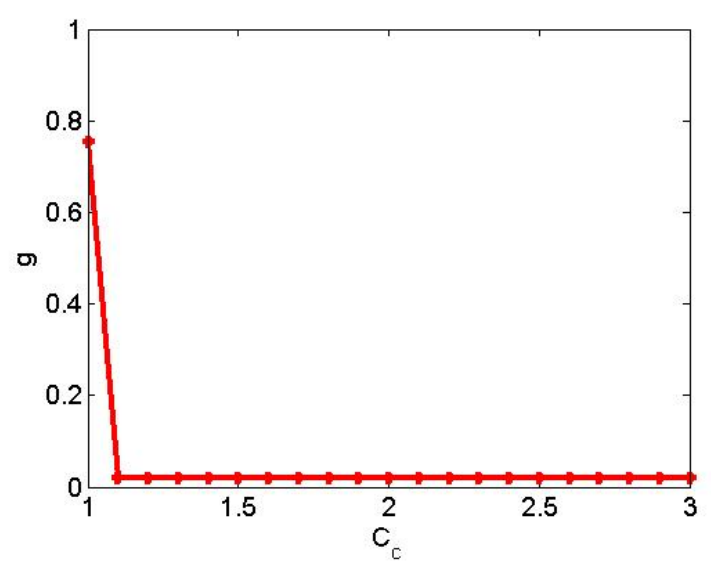

(b)

Fig. 5 Cascading failure triggered by substation 8 and 23 failure in the scale-free network is shown in subplot(a) and (b), respectively

and other nodes are consumers, we can get a directional network.

The cascading failure in the UK power grid triggered by failed generators is simulated. The plot of avalanche size $g$ versus $C_{c}$ triggered by generators 21 and 25 are shown in Fig. 8(a) and Fig. 8(b), respectively. From Fig. 8, we see that, with the increase of the tolerance parameter $C_{c}$, the avalanche size $g$ decreases. Since there is no path for consumers 16 and 20 to get power from any other generators after generator 21 failed, the avalanche size $g$ cannot settle down to 0 , no matter how large the tolerance parameter $C_{c}$ is. When generator 25 failed, there is no path for consumers 24 and 27 to get power from any other generators. So, in Fig. 8(b), the avalanche size $g$ cannot settle down to 0 , no matter how large the tolerance parameter $C_{c}$ is.

The cascading failures of UK power grid caused by substations are shown in Fig. 9. Figures 9(a) and (b) are the cascading failures triggered by the failures of 


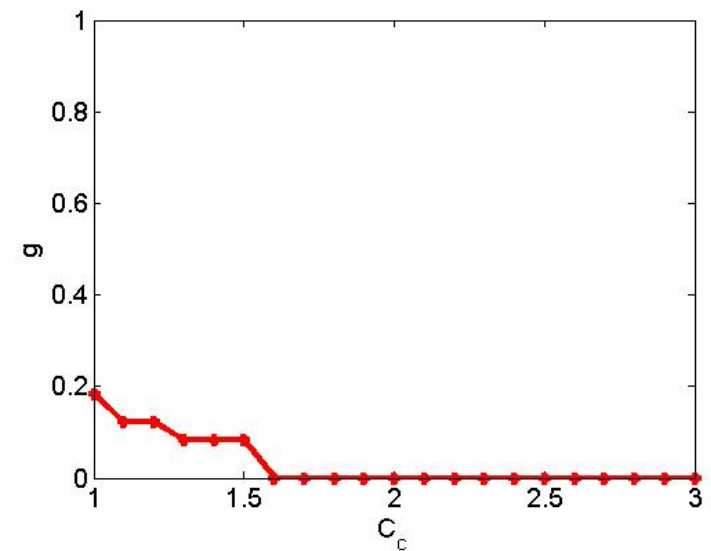

(a)

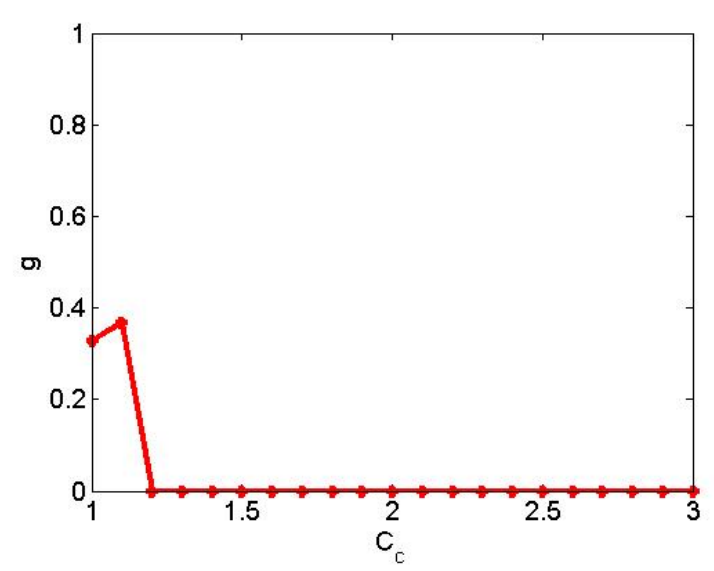

(b)

Fig. 6 Cascading failure triggered by consumers 10 and 31 failure in the scale-free network is shown in subplot(a) and (b), respectively

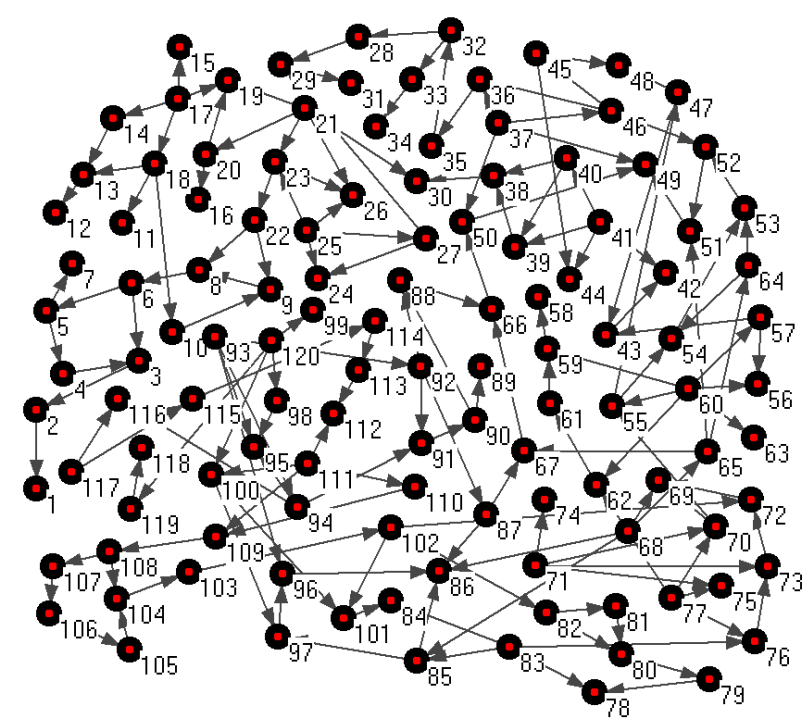

Fig. 7 UK power grid topology

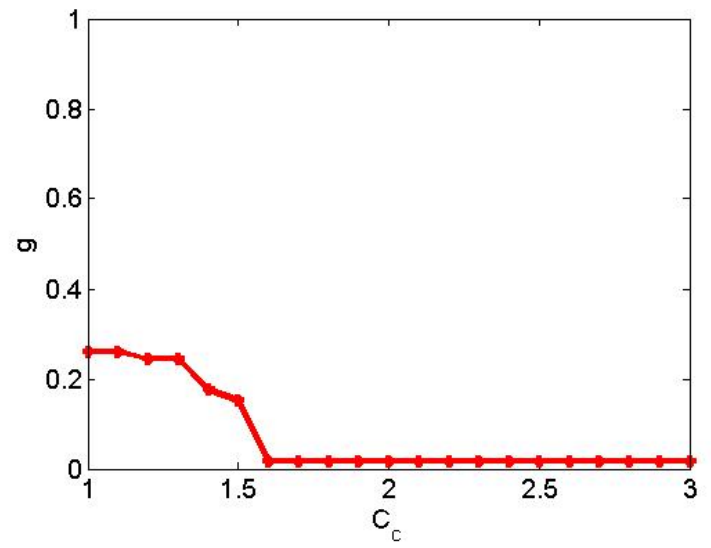

(a)

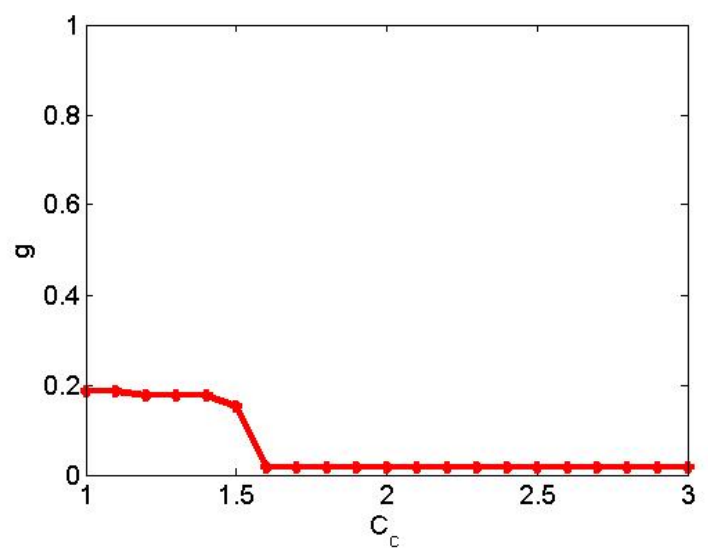

(b)

Fig. 8 Cascading failure triggered by generators 21 and 25 failure in the UK power grid is shown in subplot(a) and (b), respectively

substation 46 and 94, respectively. From Fig. 9(a), we see that, with the increase of the tolerance parameter $C_{c}$, the avalanche size $g$ decreases. When substation 46 is removed, there is no path for consumer 45 to get power from any generators. So, the avalanche size $g$ in Fig. 9(a) will not be 0 , no matter how large the $C_{c}$ is. While, in Fig. 9(b), the $g$, as a function of $C_{c}$, exhibits a non-monotonic property. Once $C_{c}$ is larger than 1.5 , the substation 94 failure does not cause cascading failure.

The cascading failures caused by consumers are shown in Fig. 10. Subplots (a) and (b) in Fig. 10 are the cascading failures triggered by the failures of consumer 19 and 28, respectively. In Fig. 10(a), the avalanche size $g$ is always 0 , since consumer 19 does not provide power to any other consumer nodes and its failure will not affect any other nodes. While, in Fig. 10(b), with the increase of the tolerance parameter $C_{c}$, the avalanche size $g$ decreases, and $g$ is 0 when $C_{c}$ is larger than 1.6. Compared to the cascading failure caused by generators 


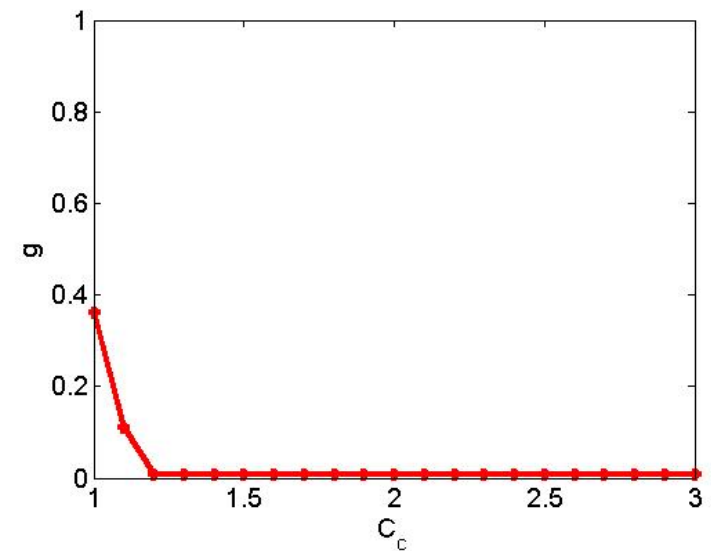

(a)

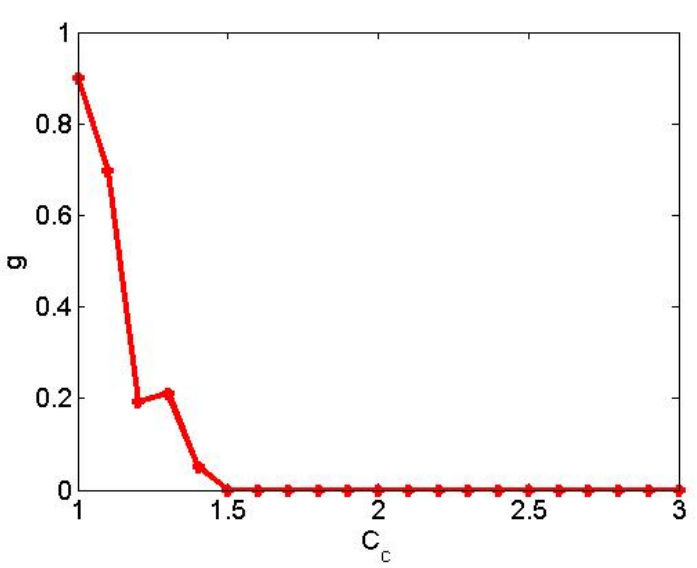

(b)

Fig. 9 Cascading failure triggered by substations 46 and 94 failure in the UK power grid is shown in subplot(a) and (b), respectively

and substations, the consumer failure will cause less effect on the scale of the cascading failure of the grid, because the load of the whole grid tends to decrease, when a consumer is removed.

4.4 Comparison of the proposed cascade failure analysis model and the model in [18]

For comparison purpose, we do the following. We compare our proposed load distribution law and node removal rule to the models in [18] with different parameters. To show the different cascade procedure using different node removal rule, we compare our proposed node removal rule to the commonly used removal rule, i.e. to remove all the overload nodes in the grid. The comparison results are shown in Figs. 11 and 12.

Figures 11 (a) and (b) show the cascading failures caused by the failed generator 22 and consumer 31 in

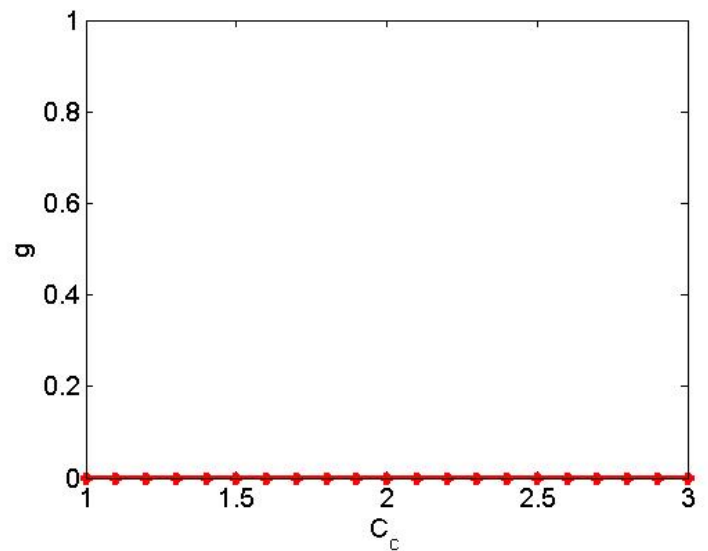

(a)

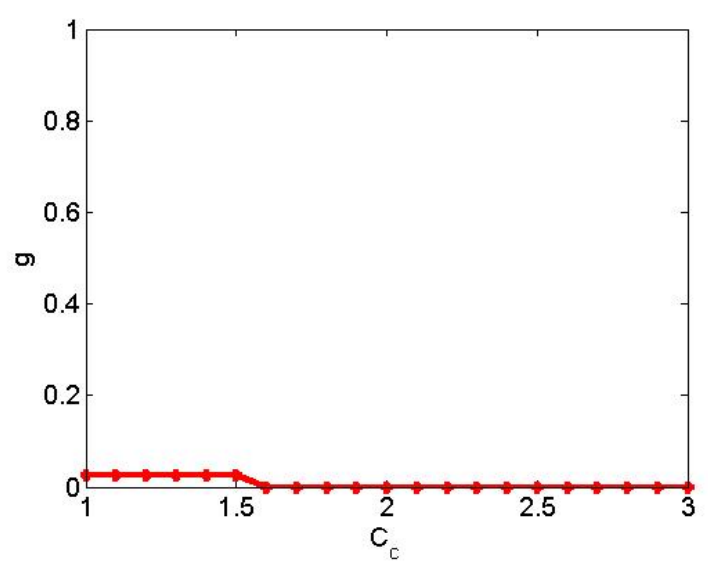

(b)

Fig. 10 Cascading failure triggered by consumers 19 and 28 failure in the UK power grid is shown in subplot(a) and (b), respectively

the scale-free network based on the two removal rules and different model parameters of the model in [18]. Figure 12 (a) and (b) show the cascading failures triggered by the failure generators 21 and 25 in the UK power grid based on the two removal rules and different model parameters of the model in [18]. From Figs. 11 and 12 , we see that using the proposed node removal rule, the avalanche size can be decreased, especially, when the tolerant parameter $C_{c}$ is small.

In addition, we simulate the cascading failures triggered by every node under the different node removal rule and get the distribution of the avalanche size as shown in Figs. 13 and 14.

Figures 13(a) and 14(a) are the avalanche size distribution using our proposed method for analyzing the 50 nodes scale free network and UK power grid, respectively. Figures 13(b) and 14(b) are the avalanche size distribution using our load distribution law and commonly used node removal rule (that removes all over- 


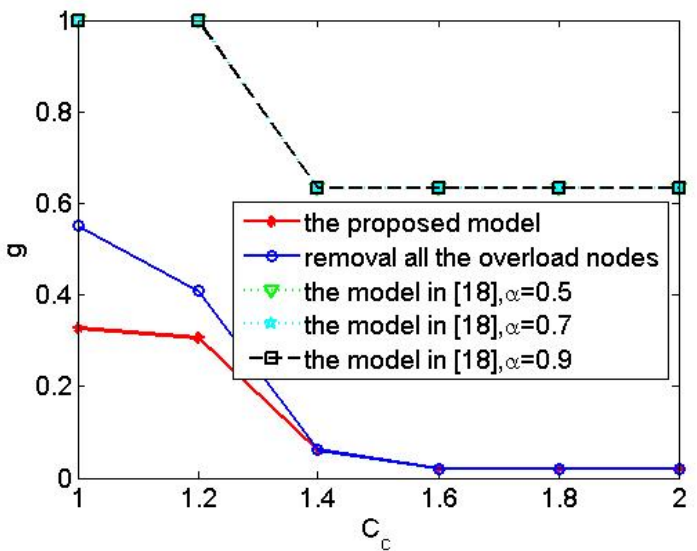

(a)

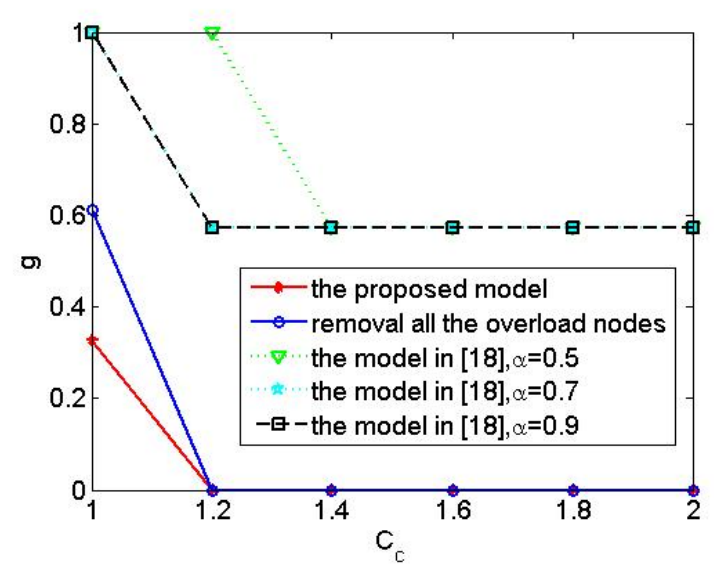

(b)

Fig. 11 Cascading failures triggered by generator 22 and consumer 31 failure in the scale-free network based on different models are shown in subplot(a) and (b), respectively

load nodes) for analyzing the 50 nodes scale free network and UK power grid, respectively. Figures 13(c) and $14(\mathrm{c})$ are the avalanche size distribution using the model in [18] with the parameter $\alpha=0.5$ for analyzing the 50 nodes scale free network and UK power grid, respectively. Figures $13(\mathrm{~d})$ and $14(\mathrm{~d})$ are the avalanche size distribution using the model in [18] with the parameter $\alpha=0.7$ for analyzing the 50 nodes scale free network and UK power grid, respectively. Figures 13(e) and $14(\mathrm{e})$ are the avalanche size distribution using the model in [18] with the parameter $\alpha=0.9$ for analyzing the 50 nodes scale free network and UK power grid, respectively.

In Figs. 13 and 14, the horizontal axis is the tolerant parameter $C_{c}$. The avalanche size distribution is given using the different bar length corresponding to the tolerant value. The black white right diagonal filled bar corresponds to a situation where one node overloads, and fails, resulting in no cascade, i.e., $g=0$. The

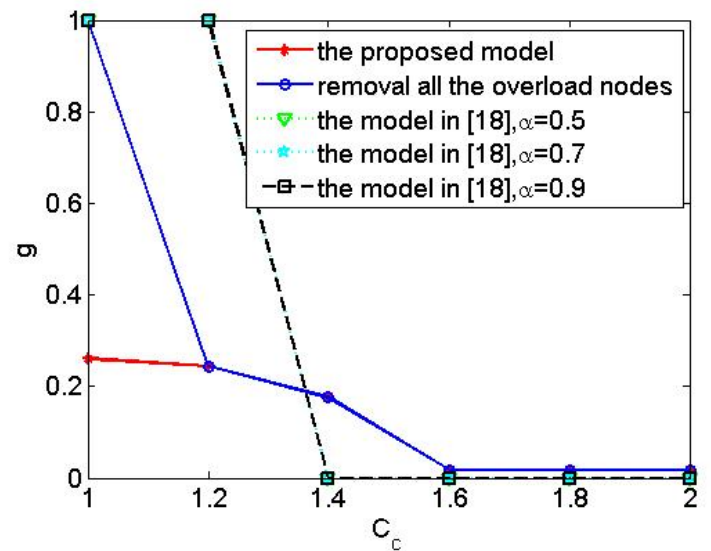

(a)

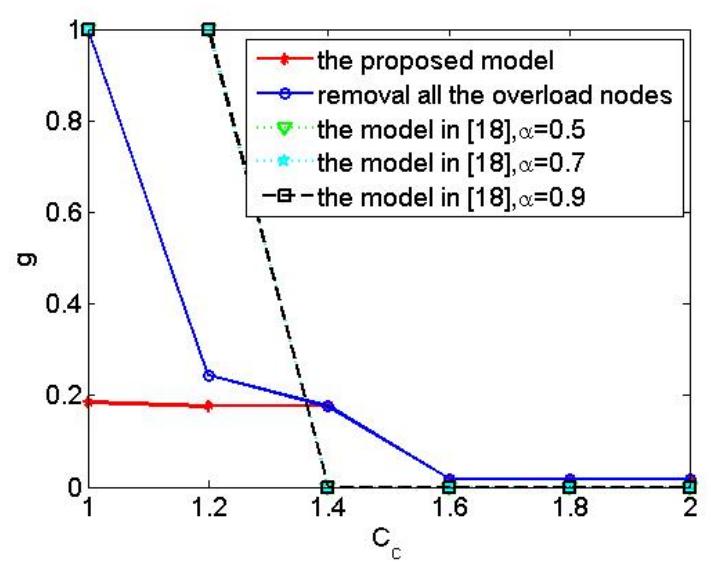

(b)

Fig. 12 Cascading failures triggered by generators 21 and 25 failure in the UK power grid based on different models are shown in subplot(a) and (b), respectively

blue horizontal line filled bar corresponds to a situation where the failure of one node followed the cascade has produced a cascade with intensity given by $0<g \leq 0.3$. The green left diagonal filled bar corresponds to $0.3<g \leq 0.5$. The orange point filled bar corresponds to $0.5<g \leq 0.7$. The brown cross filled bar corresponds to $0.7<g \leq 1$. The thickness of the bar represents the likelihood to have cascades by one node failure with that given $\mathrm{g}$ interval. For example, in Fig. 13 (a), for $C_{c}=1$ the length of black white right diagonal filled bar being 0.38 means that 38 percent of single node failure will not cause cascade failure; likewise, the length of orange point filled bar being 0.1 means that 10 percent of single node failure will cause $0.5<g \leq 0.7$.

We can see, from Figs. 13 and 14, that the proposed node removal rule has a low possibility to cause large avalanche size under the same small tolerant parameter, 

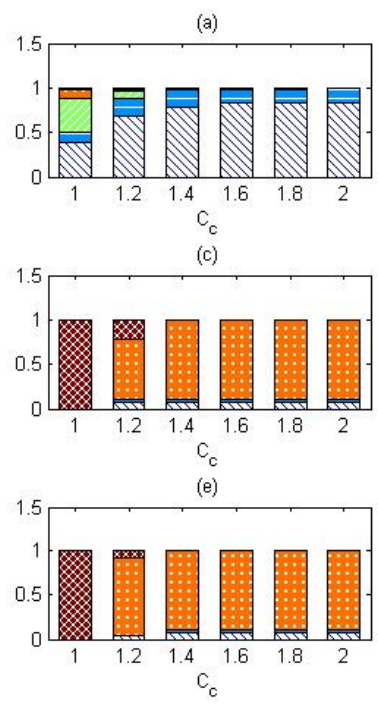

(b)

(d)
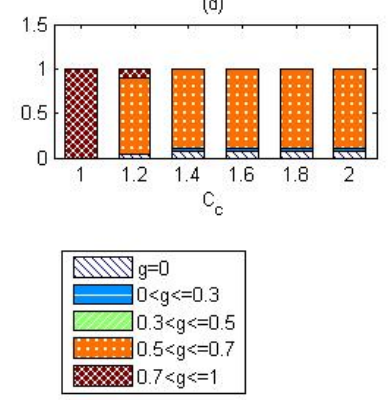

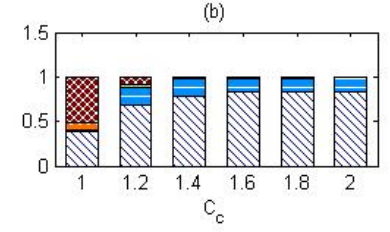

Fig. 13 The distribution of the avalanche size variation versus $C_{c}$ in the scale-free network

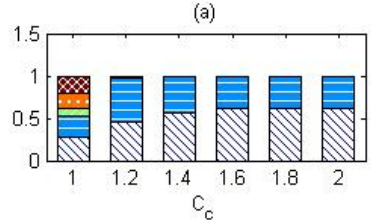

(c)

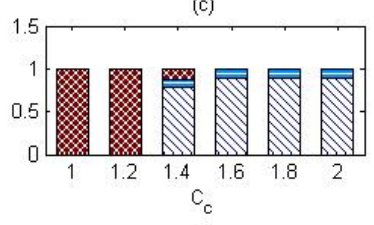

(e)

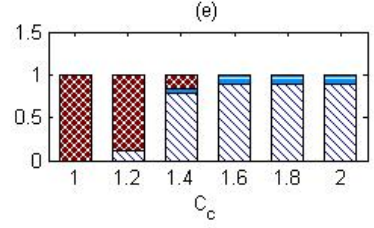

(b)

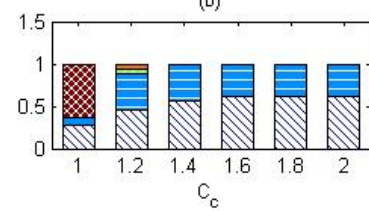

(d)
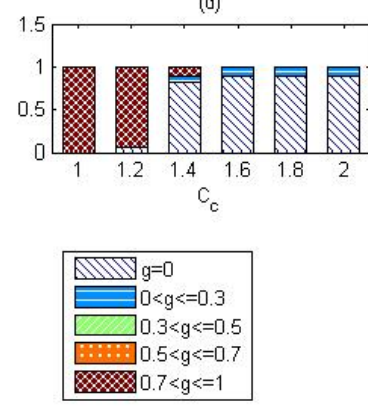

Fig. 14 The distribution of the avalanche size variation versus $C_{c}$ in the UK power grid

which helps to avoid large size cascade failure under the small tolerant parameter.

\section{Conclusion}

A new power grid model for cascading failure analysis is proposed in this work. In this model, the nodes of the power grid are divided into generators, substations, and consumers. A new load distribution law and a new node removal rule are proposed, under which the scale of cascading failure is reduced as compared to the model in [18] and to the commonly used rule of removing all the overload nodes. The proposed node removal rule does help to decrease the dangers of large scale power failure at small $C_{c}$. Our work may shed light on the tolerance parameter selection and the protective relay system design to suppress the possibility of the large scale cascade failure.

Acknowledgement The work is supported in part by NSFC (Grant no. 61172070), IRT of Shaanxi Province (2013KCT-04), EPSRC (Grant no.Ep/1032606/1).

\section{References}

1. Pahwa, S., Scoglio, Scala, A.: Abruptness of cascade failures in power grid. Sci. Rep.(2013). doi:10.1038/srep03694

2. Zheng, J.F., Gao, Z.Y., Zhao, X.M.: Modeling cascading failures in congested complex networks. Physica A 385(2), 700-706(2007)

3. Wang, J., Liu, Y.H., Jiao, Y., Hu, H.Y.: Cascading dynamics in congested complex networks. Eur. Phys. J. B 67(1), 95-100(2009)

4. Wu, J.J., Sun, H.J., Gao, Z.Y.: Cascading failures on weighted urban traffic equilibrium networks. Physica A 386(1), 407-413(2007)

5. Wu, J.J., Gao, Z.Y., Sun, H.J.: Effects of the cascading failures on scale-free traffic networks. Physica A 378(2), 505-511(2007)

6. Wu, J.J., Gao, Z.Y., Sun, H.J..: Model for dynamic traffic congestion in scale-free networks. Europhys. Lett 76(5), 787-793(2006)

7. Lizier, J.T, Prokopenko, M., Cornforth, D.J.: The information dynamics of cascading failures in energy networks. The European Conference on Complex Systems. pp.5459(2009)

8. Crucitti, P., Latora, V., Marchiori, M.: Model for cascading failures in complex networks. Phys. Rev. E 69, 045104(2004)

9. Wang, J.W.: Mitigation of cascading failures on complex networks. Nonlinear Dyn. 70(3), 1959-1967(2012)

10. Kinney, R., Crucitti, P., Albert, R., Latora, V.: Modeling cascading failures in the North American power grid. Eur. Phys. J. B 46(1), 101-107(2005)

11. Wang, B., Kim, B.J.: A high-robustness and low-cost model for cascading failures. Europhys. Lett. 78(4), 48001(2007)

12. Li, S.D., Li, L.X., Yang, Y.X., Luo, Q.: Revealing the process of edge-based-attack cascading failures. Nonlinear Dyn. 69(3), 837-845(2012)

13. Lai, Y.C., Motter, A.E., Nishikawa, T.: Attacks and cascades in complex networks. Lecture Notes in Physics 650, 299-310(2004)

14. Wu, Z.X., Peng, G., Wang, W.X., Chan, S., Wong, E.W.M.: Cascading failure spreading on weighted heterogeneous networks. J. Stat. Mech. P05013(2008)

15. Zhang,G.D., Li,Z., Zhang,B., Halang, W.A.: Understanding the cascading failures in Indian power grids with complex networks theory. Physica A 392(15), 3273$3280(2013)$

16. Wang, J.W., Rong, L.L., Zhang, L., Zhang, Z.Z.: Attack vulnerability of scale-free networks due to cascading failures. Physica A 387(26), 6671-6678(2008) 
17. Wang, J.W., Rong, L.L.: Effect Attack on scale-free networks due to cascading failures. Chin. Phys. Lett. 25(10), 3826-3829(2008)

18. Wang, J.W., Rong, L.L.: Cascade-based attack vulnerability on the U.S. power grid. Saf. Sci. 47(10), 13321336(2009)

19. Wang, J.W., Rong, L.L.: A model for cascading failures in scale-free networks with a breakdown probability. Physica A 388(7), 1289-1298(2009)

20. Wang, W.X., Lai, Y.C.: Abnormal cascading on complex networks. Phys. Rev. E 80, 036109(2009)

21. Bao, Z.J., Cao, Y.J., Wang, G.Z., Ding, L.J.: Analysis of cascading failure in electric grid based on power flow entropy. Phys. Lett. A 373(34), 3032-3040(2009)

22. Simonsen, I., Buzna, L., Peters, K., Bornholdt, S., Helbing, D.: Transient dynamics increasing network vulnerability to cascading failures. Phys. Rev. Lett. 100, 218701(2008)

23. Filatrella, G., Nielsen, A.H., Pedersen, N.F.: Analysis of a power grid using a Kuramoto-like model. Eur. Phys. J. B 61(4), 485-491(2008)

24. Carareto, R., Baptista, M.S., Grebogi, C.: Natural synchronization in power-grids with anti-correlated units. Commun. Nonlin. Sci. Numer. Simulat 18(4), 10351046(2013)

25. Sakaguchi, H., Matsuo, T.: Cascade Failure in a Phase Model of Power Grids. J. Phys. Soc. Jpn 81(7), 074005(2012)

26. Guo Chen, Zhao Yang Dong, David J.Hill, Guo Hua Zhang.: An improved model for structural vulnerability analysis of power networks. Physica A 388(19), 42594266(2009)

27. Elmore, W.A.: Protective relaying: theory and applications. CRC Press, New York(2003)

28. He, J.L.: Power system protective relay theory. China Electric Power Press, Beijing(2010)

29. Witthaut, D., Timme, M.: Braesss paradox in oscillator networks, desynchronization and power outage. New J.Phys. 14(8), 083036(2012) 